\title{
Recalled Positive Influences within Life-Story Interviews and Self-Reported Generative Concern in German Older Adults: The Moderating Role of Extraversion
}

\author{
Holger Busch ${ }^{1} \mathbb{D} \cdot$ Jan Hofer ${ }^{1} \mathbb{D}$ \\ Accepted: 8 June 2021 / Published online: 22 June 2021 \\ (c) The Author(s) 2021
}

\begin{abstract}
Previous research has shown that recalling positive influences in one's life story correlates with generative concern. Given findings that not everyone benefits from generative efforts uniformly, however, the present study tested if extraversion moderates this relation. In total, 147 older German adults (59 through 83 years) recalled positive influences in their lives in an interview session and provided self-report questionnaire data on their generative concern (Loyola Generativity Scale), generative behavior (Generative Behavior Checklist), and extraversion (Mini-IPIP scales). Results from a moderated mediation model indicate that recalled positive influences related to generative concern but not generative behavior. Moreover, extraversion did indeed moderate between recalled positive influences and generative concern in that the relation was significantly positive for medium and high extraversion. The findings suggest that what people learn from generative role models is generative concern rather than generative behavior. They also suggest a twofold role of extraversion for generativity: It has been found to be a predictor of generativity but also affects what people gain from others' generative efforts.
\end{abstract}

Keywords Generativity $\cdot$ Generative concern $\cdot$ Generative behavior $\cdot$ Extraversion $\cdot$ Positive influences $\cdot$ Life story

In his lifespan developmental theory, Erikson (1963) introduced the term generativity to denote individuals' desire to help younger generations thrive (Kotre, 1984). This can be achieved in various social domains such as the family (i.e., as parents, see Peterson et al., 1997, or as grandparent, see Moore \& Rosenthal, 2014), the work place (Zacher et al., 2011), or politics (Peterson \& Duncan, 1999). As such, generativity is highly socially beneficial (McAdams, 2013). Moreover, generativity is associated with well-being (e.g., Grossman \& Gruenewald, 2020; Hofer et al., 2008). While generally primarily associated with middle adulthood, generativity also plays a major role in the lives of older adults (Erikson \& Erikson, 1997): For example, when interviewed, older people describe generativity as a core aspect of successful aging (Fisher, 1995). Moreover, generativity predicts psychological functioning and well-being in older samples (e.g., Busch et al., 2018; Gruenewald et al., 2012; Versey

Holger Busch

busch@uni-trier.de

1 Department of Psychology, Developmental Psychology, Trier University, 54286 Trier, Germany et al., 2013). Thus, given its individual and societal benefits, it is important to study how generativity develops. The present study did so by (a) examining how recalled positive influences in the life stories of older participants relate to their generative concern and behavior, and (b) testing if participants' trait extraversion moderates the relationship between recalled positive influences and generative concern.

\section{The Development of Generativity}

Apart from a genetic component (Faßbender et al., 2019), research has identified family factors that contribute to an individual's generativity development. For example, parents' authoritative parenting style is positively associated with their adult offspring's generativity (Peterson et al., 1997). Similarly, parents' emphasis on caring predicts their adolescent offspring's generativity across a four-year interval (Frensch et al., 2007). Moreover, when generative parents teach their adolescent children values they make more use of certain story characteristics (i.e., interactivity, specificity) which in turn relate to their offspring's generativity (Pratt et al., 2008). 
However, there is abundant evidence that generativity also is learnt outside the family. For example, taking care of one's friends is a developmental precursor of generativity in adolescents (Lawford et al., 2013). In general, generativity correlates with social support from family and non-family sources (Grych et al., 2020; Hart et al., 2001), suggesting that generativity might be learnt particularly when oneself has been the recipient of somebody else's generative efforts (Kessler \& Staudinger, 2007). Results from an early childhood development program corroborate this conclusion in that its participants scored higher on generativity as adolescents than comparison youth (Love et al., 2013).

In fact, young men who had a mentor reported more generativity as a midlife adult than those who had not (Westermeyer, 2004). In a similar vein, the number of mentor figures in young adulthood correlates positively with midlife generativity motivation (Peterson \& Stewart, 1996). Jones and McAdams (2013) reported a comparable finding but pointed out that the positive relation is found in Euro-American but not African-American participants. More recently, Busch and Kranz (2021) showed that received generativity contributes to provided generativity in gay men by fostering their gay identity affirmation. It is important to emphasize that in these studies, mentor figures have not been understood in terms of formal mentors (as, for example, in official career mentoring programs in companies) but as older people with more life experience who passed on their knowledge and experiences to the participant. In sum, research supports the notion that receiving generative care - whether from someone inside or outside the family - is conducive to an individual's generativity (McAdams, 2013).

Apart from the agreement regarding this broad conclusion, however, the studies referenced above differ in how they have approached generativity, that is, in terms of generative concern or generative behavior. In their generativity model, McAdams and de St. Aubin (1992) defined generative concern as a conscious "concern for and interest in promoting the next generation" (McAdams et al., 1998, p. 20). It, thus, describes a general inclination towards generativity in that individuals cherish the idea of contributing to the well-being and thriving of coming generations. Generative concern predicts corresponding generative behavior in middle and old adult samples (e.g., Cox et al., 2010; Hofer et al., 2016; for an overview, see, e.g., McAdams, 2013), which is the behavioral realization of a generative inclination. As delineated above, people can show generative behavior in a wide variety of contexts.

So, while some studies propose that positive influences foster the development of generative concern (Grych et al., 2020; Hart et al., 2001; Jones \& McAdams, 2013; Lawford et al., 2013), others suggest that positive influences have an effect on generative behavior (Busch \& Kranz, 2021; Kessler \& Staudinger, 2007; Westermeyer et al., 2004). To disentangle these two propositions, we tested a mediation: We hypothesized that positive influences relate primarily to generative concern (Jones \& McAdams, 2013). Generative concern, then, relates to generative behavior (McAdams, 1992, 1998). This reasoning does not rule out, however, that positive influences might be associated with both, generative concern and generative behavior. This, then, would show in a combination of a direct effect of positive influences on generative behavior and an indirect one through generative concern.

\section{Generativity and Extraversion}

Is it to be expected that everybody will benefit from mentoring in the same way, however? In general, the personality trait of extraversion seems to modulate the effect that interventions have on participants. For example, a life-review intervention was more successful in decreasing depressive symptoms in more extraverted individuals (Korte et al., 2012). Similarly, a humor-based intervention in midlife women had a more positive effect on their happiness when they were more extraverted (Wellenzohn et al., 2018). Thomas et al. (2021) reported that extraversion affected how much adolescents benefited from an intergenerational encounter group in terms of their self-concept clarity. This result is of particular relevance to the present study as it indicates that generative efforts may be received differentially based on the recipients' trait of extraversion. ${ }^{1}$

Marr et al. (2020) suggested that extraverted individuals might benefit more from less structured interventions that provide more opportunity for social interactions. This might be due to some characteristics of extraverts: For example, extraverts maintain and nurture their social relationships, which might give their mentor figures more opportunity to offer more advice (Dougherty et al., 2007). Moreover, they are more likely to ask for social support when they find themselves in a stressful situation than introverts (Watson \& Hubbard, 1996), so that mentor figures can give advice that is more specific. This is in line with findings that extraversion relates to selfdisclosure (Carpenter \& Freese, 1979; Sun et al., 2020), which mentor figures might reciprocate by relating some of their life

\footnotetext{
$\overline{1}$ To be more specific, Thomas et al. (2021) did not find any moderation effect for conscientiousness, neuroticism, or openness to experience. They did find, however, an additional moderation for agreeableness, arguing that extraversion and agreeableness are most closely linked to social behavior and experience (Tov et al., 2016). Based on these findings, we did not consider conscientiousness, neuroticism, or openness to experience in the present research. Furthermore, no analyses on agreeableness are presented as the corresponding measure featured an internal consistency in the present study that was below what is considered acceptable (i.e., <.6; Ursachi et al., 2015).
} 
experiences. Thus, the effects of informal mentoring should covary with participants' extraversion.

Extraversion is a well-documented correlate of generativity (e.g., Blatný et al., 2019; Cox et al., 2010; Peterson et al., 1997). Extraverts find it easier to interact with others than introverts do (McCrae \& Costa, 2003), which should facilitate generativity. The focus of the present research, however, is less on the relation between generativity and extraversion itself, but rather on how extraversion shapes experiences of receiving generativity so that they become conducive to generativity development.

\section{The Present Research}

Previous research has shown that recalled positive influences in one's life story relate to generativity development. Specifically, recalling such influential figures outside the family, for example, mentors, was a predictor of generative concern (Jones \& McAdams, 2013). That is, people who benefit from some positive influence's generative efforts learn to care for future generations and later realize this concern in generative behavior. This learning opportunity presented by the positive influence may be used differentially, however: In an intergenerational exchange program, it was particularly the extraverted adolescents whose self-concept clarity increased (Thomas et al., 2021). Thus, extraversion might moderate how much people benefit from positive influences in terms of their own generativity development.

Drawing on these findings, we tested a moderated mediation: Specifically, we hypothesized that recalled positive influences are positively associated with generative concern. This association, however, was hypothesized to be moderated by extraversion: Because they may feel more comfortable in unstructured social interactions, extraverts should show behavior that elicits more advice and sharing of life experiences from their positive influences. Thus, they should benefit more from positive influences - that is, the relationship between positive influences and generative concern should be closer when extraversion is high. Finally, because generative concern facilitates generative behavior, we hypothesized that recalled positive influences are linked indirectly to generative behavior through generative concern. The inclusion of generative behavior in the model also allowed us to test if positive influences primarily predict generative concern or generative behavior as well.

\section{Methods}

\section{Procedure}

The present data are part of a cross-cultural study on successful aging (e.g., Busch et al., 2018; Hofer et al., 2016).
As not all instruments were employed at all cultural sites, the present study focused exclusively on the German sample. Hofer et al. (2016) also reported analyses on the relation between generative concern and generative behavior in the present sample. As, however, the inclusion of generative behavior in the present study offered the opportunity to test if recalled positive influences relate to generative concern and/or generative behavior, we think this is justified.

The study was announced in a local newspaper report, which featured a contact for those interested in participating. Participants were invited to university premises for data collection. A trained student assistant was present to respond to all questions if any should arise. First, participants worked through a set of questionnaires (among them the instruments for generative concern and behavior, see below). Then, they provided sociodemographic information (e.g., age, gender). Finally, some participants were asked some questions in an interview form. This was not possible for all participants, for example, because of conflicting time schedules. One of the interview questions referred to positive influences in one's life story (see below).

All participants were informed about the purposes of the study and provided informed consent. Participant codes were used to match questionnaire and interview data without endangering participants' anonymity. In general, the study was conducted in accordance with the Declaration of Helsinki. Participants received financial compensation for their participation.

\section{Participants}

For the present analyses, data from 147 participants were used (73 females, 74 males). They ranged from 59 through 83 years in age $(M=68.03, S D=5.90)$. Originally, a minimum age of 60 years had been set as inclusion criterion, but three participants aged 59 took part, and we decided against discarding their data. Eleven participants who had taken part in the interview session produced missing data in the questionnaire, here defined as more than $10 \%$ of items of a given scale unanswered. One participant's data were discarded because of multivariate outliers. Seven additional participants did not provide any response to the interview question although they had initially consented to participate in the interview session.

\section{Measures}

\section{Positive Influences}

Participants were asked about the positive influences that have had a long-lasting impact on their lives. The question was composed to correspond to the "III. Important Persons" section of the 1995 version of the Life-Story Interview (as 
Table 1 Descriptive statistics of and correlations among positive influences, generative concern, generative behavior, and extraversion

\begin{tabular}{llllllll}
\hline & $M$ & $S D$ & 1 & 2 & 3 & 4 \\
\hline 1 & Positive influences & 0.830 & 0.961 & - & & \\
2 & Generative concern & 1.659 & 0.400 & $.204 *$ & - & \\
3 & Generative behavior & 0.576 & 0.193 & .061 & $.504 * * *$ & - & \\
4 & Extraversion & 1.922 & 0.632 & -.070 & $.283 * * *$ & .147 & - \\
\hline$* p<.05 ; * * p<.01 ; * * * p<.001$ & & & & &
\end{tabular}

described in McAdams et al., 1997, and found in https:// sites.northwestern.edu/thestudyoflivesresearchgroup/instr uments/). Specifically, the question was: "In everybody's life there is a multitude of people that play a role in the life story. Some people play only a minor role, but others play a major role and have a great influence in one's life. Now I would like to learn a bit about those people that have had a big influence on your life. Looking back over your life story, please identify those persons that have or have had a great positive influence on your life story. Please describe these persons and the way in which they have had a positive impact on your life."

Participants' responses were audiotaped, transcribed in verbatim, and subsequently coded for the number of positive influences that each participant named. If participants made clear that (a) someone had a long-lasting influence on them and (b) that influence had a positive valence for the participant, the respective person was coded as positive influence. That is, the positive influences score reflects the number of individuals that a participant mentioned who meet the aforementioned conditions of a long-lasting positive influence. Thus, a participant's positive influence score is 1 , if they talked about a single person, even if they might give more than one example of how that person had influenced them. In coding, we focused on extra-familial individuals (cf. McAdams et al.'s, 1997, helper category) so that our positive influence score corresponds closely to Jones and McAdams' (2013) positive teacher/mentor influence category. Three examples are given in Appendix A (abbreviated for the sake of readability).

According to recommendations (Syed \& Nelson, 2015), the first author and two coders worked through 20 interviews to discuss the exact scoring procedure. Then, the two coders coded 30 interviews to determine interrater reliability. With $\kappa=.781, p<.001$, reliability was good (cf. Syed \& Nelson, 2015), so that both coders then continued to score the remaining interview responses independently. In case of uncertainty, coders met and agreed on the final score.

\section{Generative Concern}

The Loyola Generativity Scale (LGS; McAdams \& de St. Aubin, 1992) features 20 statements that reflect an individual's concern for the coming generations. For each statement (e.g., "I have important skills that I try to teach others"), participants indicated how much it applies to them $(0=$ statement never applies to you; $3=$ statement applies to you very often or nearly always). Cronbach's Alpha was .713.

\section{Generative Behavior}

Consisting of 40 generative activities (e.g., "did volunteer work for a charity"), the Generative Behavior Checklist (GBC; McAdams \& de St. Aubin, 1992) asks participants how frequently they have performed each generative act during the last two months $(0=$ not at all; $2=$ more than once $)$. Cronbach's Alpha was .823.

\section{Extraversion}

Donnellan et al. (2006) developed the Mini-IPIP scales to measure the Big Five personality traits in an economic way. Each trait is measured with four items. For extraversion, the items read, "I am the life of the party," "I don't talk a lot" (reverse scored), "I talk to a lot of different people at parties," and "I keep in the background" (reverse scored). Participants gave their assessment as to how well the statement describes them $(0=$ not at all true; $4=$ very true $)$. Cronbach's Alpha was .646.

\section{Results}

Table 1 presents descriptive statistics on positive influences, generative concern, generative behavior, and extraversion. Participants named up to four positive influences. As, however, there were only three participants who listed four positive influences and statistically, these scores turned out to be extremes, we set these three participants' positive influences score to three. Rerunning analyses with unchanged positive influences scores yielded results that are in line with those presented below. Corresponding output is available in OSF (https://osf.io/6yvbr).

Positive influences correlated significantly only with generative concern. Extraversion, too, significantly related to generative concern; its correlation with generative behavior marginally failed to reach significance, $r=.147$, $p=.077$. Finally, there was a significant correlation between 


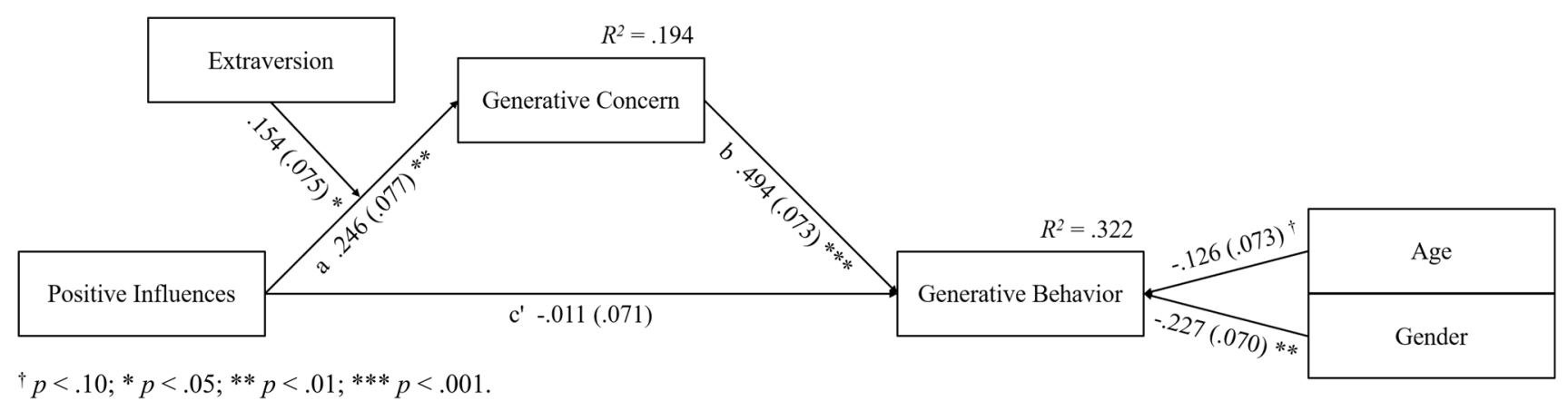

Fig. 1 Generative concern mediates the relation between positive influences and generative behavior, with extraversion moderating the path from positive influences to generative concern. Path coefficients

generative concern and generative behavior. Age was significantly negatively associated with generative concern, $r=-.218, p=.008$, and generative behavior, $r=-.232$, $p=.005$. There was no gender difference for generative concern, $t(145)=-.803, p=.423$, but for generative behavior, $t(145)=2.383, p=.018$, with female participants, $M=0.613$, $S D=0.199$, reporting more generative behavior than male participants, $M=0.539, S D=0.182$. Thus, both age and gender were included as covariates in the moderated mediation analysis. All variables were $\mathrm{z}$-standardized before the analysis was run.

To test the moderated mediation hypothesis, we employed PROCESS model 7 (Hayes, 2018), which defines that the moderator affects the path between predictor and mediator (path a) but not the path between mediator and criterion (path b; path terminology according to Baron \& Kenny, 1986). Indirect effects were interpreted as significant when the bias-corrected bootstrapped $95 \%$ confidence intervals (95\% CIs; 10,000 bootstrap samples) did not include zero.

With $N=147$, the sample size fulfill requirements for detecting a simple mediation effect at power $=.80$ for a small-to-medium path a and a medium path b (Fritz \& MacKinnon, 2007). Monte-Carlo simulation yielded a .71 power for the detection of a simple mediation (Schoemann et al., 2017); a corresponding power analysis for moderated mediation does not exist yet.

Figure 1 illustrates the moderated mediation and provides unstandardized coefficients $(B \mathrm{~s})$, along with corresponding standard errors (SEs) in parentheses. As can be seen, the direct effect (c') of positive influences on generative behavior was not significant, whereas path $a$, path $b$, and the interaction term of positive influences * extraversion were.

The index of moderated mediation was significant, $B=0.076$, BootSE $=0.040,95 \% C I[0.003,0.158]$, indicating that the indirect effects at different levels of the moderator extraversion differed from each other (Hayes, 2015). $R^{2}$ change for the interaction term was $.024, F(1,143)=4.258$, $p=.041$. While the indirect effect of positive influences on are unstandardized regression weights $(B \mathrm{~s})$ with corresponding standard errors $(S E \mathrm{~s})$ in parentheses. Gender was coded $0=$ female and $1=$ male. ${ }^{\dagger} p<.10 ; * p<.05 ; * * p<.01 ; * * * p<.001$

generative behavior was nonsignificant when extraversion was low, it was significant when extraversion was medium or high (see Table 2 for direct and indirect effects). Low, medium, and high extraversion corresponds to scores at one standard deviation below the mean, at the mean, and one standard deviation above the mean, respectively.

The moderation is illustrated in Fig. 2, which shows the graphs for the relationship between positive influences (given on the $\mathrm{x}$-axis at one standard deviation below the mean, the mean, and one standard deviation above the mean) and generative concern (given on the y-axis in standard deviation units with 0 equaling the mean) for the three levels of extraversion delineated above (i.e., one standard deviation below the mean, the mean, and one standard deviation above the mean). Note that extraversion positively related to generative concern, $B=0.281$, BootSE $=0.077, p<.001$.

In an additional analysis, we added the interview word count (for a similar procedure see, e.g., McAdams \& Guo, $2015)$ to the model because positive influence scores related to interview length, $r=.171, p=.039$. This addition,

Table 2 Direct and indirect effects of positive influences on generative behavior through generative concern with the relation between positive influences and generative concern moderated by extraversion

\begin{tabular}{lrrr}
\hline & $B$ & $S E$ & $95 \%$ CI \\
\hline $\begin{array}{l}\text { Direct effect } \\
\text { Positive influences } \rightarrow \text { Generative }\end{array}$ & -.011 & .071 & {$[-.152, .130]$} \\
$\quad$ behavior & & & \\
Indirect effect: Positive influences $\rightarrow$ Generative concern $\rightarrow$ Gen- \\
$\quad$ rative behavior at different levels of extraversion \\
Low extraversion $(-1 S D)$ & .046 & .062 & {$[-.069, .177]$} \\
Medium extraversion $( \pm 0 S D)$ & .122 & .046 & {$[.037, .219]$} \\
High extraversion $(+1 S D)$ & .198 & .060 & {$[.092, .326]$} \\
\hline
\end{tabular}

Scores for the moderator variable extraversion are given at one standard deviation below the mean (low extraversion), at the mean (medium extraversion), and one standard deviation above the mean (high extraversion), respectively 


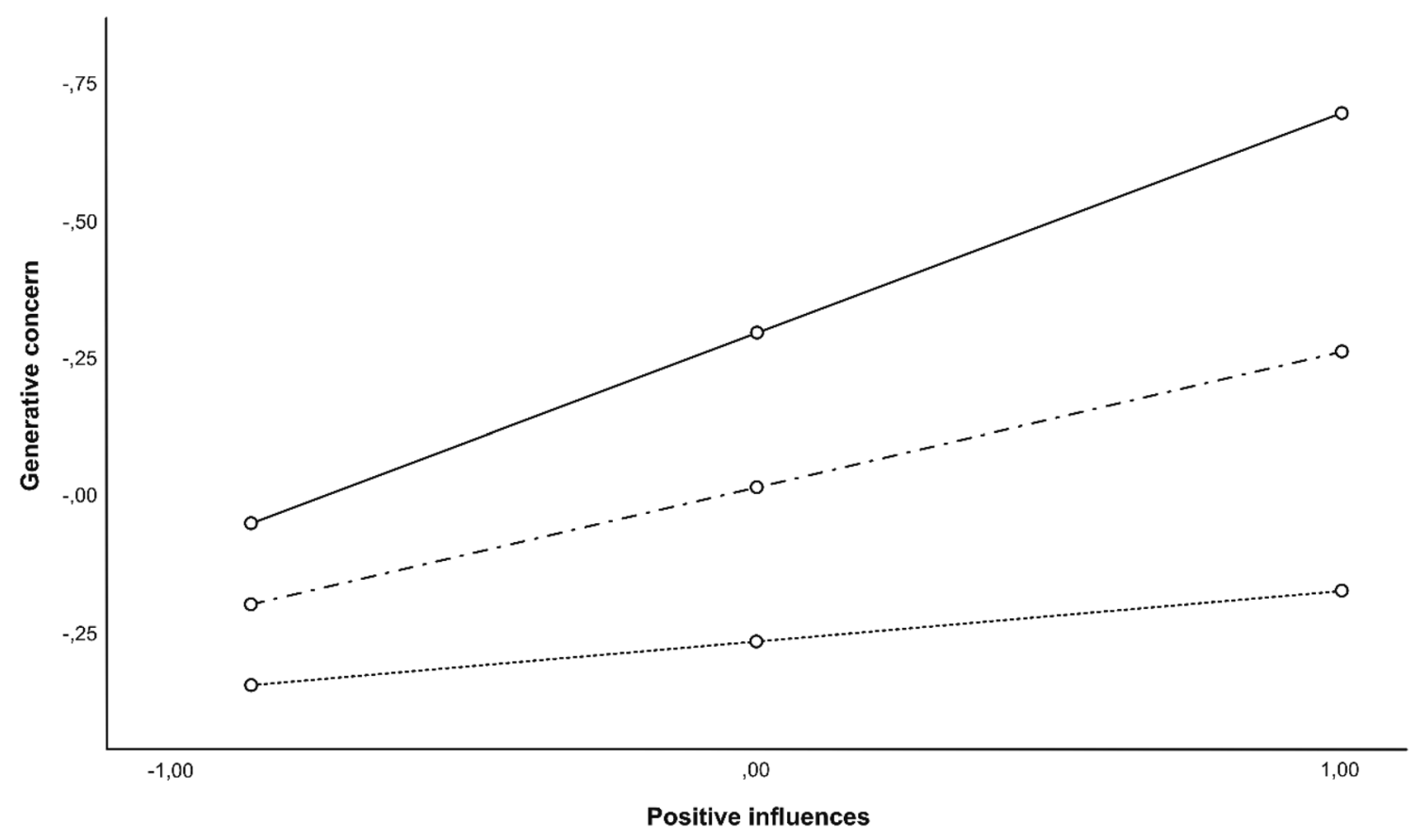

Fig. 2 The moderating effect of extraversion on the relation between positive influences and generative concern. Solid line: high extraversion (one standard deviation above the mean); dashed line: medium extraversion (the mean); dotted line: low extraversion (one standard deviation below the mean). On the $\mathrm{x}$-axis, scores for positive influences are given at one score below the mean, at the mean, and one

however, did not change the results as presented here. Finally, rerunning analyses without any of these covariates did not substantially affect results either. All corresponding outputs are available in OSF (https://osf.io/6yvbr).

\section{Discussion}

Based on previous research on the relation between positive influences and generativity, we hypothesized that recalled positive influences in participants' life story would relate to their generative concern (Jones \& McAdams, 2013). Given findings on how extraversion moderated the effects of generative efforts (Thomas et al., 2021), however, we additionally hypothesized that the relation between positive influences and generative concern would be closer when extraversion is high. Drawing on findings on the relation between generative concern and generative behavior (e.g., McAdams et al., 1998), we added the latter to the model so that we tested a moderated mediation: Specifically, positive influences related to generative concern, but extraversion did indeed moderate this link. Positive influences had a solely indirect effect on generative behavior through generative concern, but no direct effect emerged. standard deviation above the mean; note, however, that for participants who did not report a single positive influence in their life stories, the z-standardized score was -.864 . Scores for generative concern are also given in terms of standard deviations (i.e., 0 equals the mean)

Thus, in the present study, we replicated the positive relation between recalled positive influences outside the family context and generative concern (Jones \& McAdams, 2013). This indicates that, on the one hand, role models and mentors can express their generativity by taking care of future generations (Marsaglio, 2008), and on the other hand, the recipients of this care do actually benefit from this care in terms of their own generativity development (Busch \& Kranz, 2021). Moreover, we could specify what aspect of generativity recalled positive influences affect: The fact that positive influences related to generative concern, but not generative behavior, suggests that, in the long run, people learn a positive attitude toward generativity from those whose generative efforts they benefitted from. Imitation learning might be more relevant to short-term learning of prosocial behavior (Kessler \& Staudinger, 2007), but for long-term generativity development, the internalization of generative attitudes might be required. This is in line with the generativity model that posits generative concern as the first instance in the sequence towards actual generative behavior (McAdams et al., 1998; McAdams \& de St. Aubin, 1992). We would, thus, expect that were studies demonstrating an association of positive influences and generative behavior (e.g., Busch \& Kranz, 2021) complemented with 
a generative concern measure, the same indirect effect as found in the present study would emerge.

It is noteworthy that McAdams et al. (1997) forwarded seemingly contradictory results to the present ones: Using a definition of positive influences that corresponds to the one employed in the present study, they did not find any difference between more and less generative individuals in how many positive influences they had experienced. How can this finding be reconciled with the significant relation between positive influences and generative concern here and in Jones and McAdams (2013); McAdams et al. (1997) predominantly looked at childhood experiences because they were interested in early influences on generativity development (see also McAdams, 2013). This difference might well account for divergent results because positive influences might also appear later in life, as the examples in Appendix A illustrate. Thus, generativity development seems to receive impulses in childhood (Love et al., 2013), adolescence (Lawford et al., 2013), and adulthood (Erikson, 1963).

The relation between recalled positive influences and generative concern, however, was qualified by an interaction with extraversion. This indicates that recipients' personality affects generativity development in two ways: One way is that due to feeling more comfortable in social interactions, extraverts are more likely to engage in generativity (Blatný et al., 2019; Cox et al., 2010). This reflects in the positive relation extraversion had with generative concern in the present data. The other way is that other people's generative efforts may affect people differentially depending on their extraversion (Thomas et al., 2021). This way reflects in the moderating effect extraversion exerted on the link between recalled positive influences and generative concern.

At this point, it seems advisable to contextualize the current results with respect to participants' age. Jones and McAdams (2013) examined positive influences and generativity in participants in their mid-fifties. The present study complements this by including older participants. Together with other findings on received and provided generativity (Busch \& Kranz, 2021), this indicates that supportive individuals can foster people's generativity development across adulthood. In a similar vein, the moderating effect of extraversion on the benefits of broadly defined interventions has occurred in adolescents (Thomas et al., 2021) as well as older adults (Korte et al., 2012). This suggests that extraversion might increase the effects of unsystematic social interventions across adulthood, particularly given the stability of extraversion across the adult lifespan (McCrae \& Costa, 2003).

Why did extraverts in the present sample benefit more from positive influences in terms of their generative concern development? One potential answer is that extraverts are perceived as more involved (e.g., interested in what the other has to say) by social interaction partners in unstructured situations (Eaton \& Funder, 2003). Hence, extraverts might elicit more care or mentoring from their positive influences because they tend to create more positive interactions with them, thereby motivating influential figures to invest more in their relationship (cf. the finding that perceived rejection of generative efforts reduces generativity, Tabuchi et al., 2015). That is, whereas the number of positive influences did not significantly relate to extraversion, the intensity of generative efforts by the positive influence might well do. Future research might test this explanation by additionally assessing positive influence intensity, for example, by probing for the degree of self-disclosure of the positive influence or the readiness to seek for social support with the positive influence figure.

The present results do not mean that extraversion must necessarily be beneficial for intervention effects in the broadest sense. For example, extraverted children showed less benefit from an anti-aggression training (Stoltz et al., 2012). In this particular example, the authors attributed this to the reduced impulse control component of extraversion. This leads us to a limitation of the present study: Extraversion is a multifaceted trait and some may be more closely related to generativity than others (Cox et al., 2010). The extraversion measure employed here is a brief instrument, which is designed to measure traits as broadly as possible (Donnellan et al., 2006). However, the brief scales seem to capture warmth and gregariousness better than, for example, activity level and excitement seeking. Thus, different extraversion facets may play a role in moderating effects of social influences or interventions. For that reason, future research might try to replicate the present results with a fuller extraversion assessment tool. Besides, other traits might be worth considering in this context, particularly agreeableness (Thomas et al., 2021; Tov et al., 2016). Again, however, a detailed look at specific facets would be required. For example, the trust component of the agreeableness trait would also be a candidate for a moderating influence on the link between positive influences and generative concern.

\section{Limitations, Outlook, and Practical Implications}

Above, we have already discussed a limitation with respect to the extraversion measure. The use of a more comprehensive measure might also have the advantage of an increased internal consistency, which in the present sample was low but still in the acceptable range of above 0.6 (Ursachi et al., 2015). Another limitation concerning assessment refers to the positive influences: In the interview question, we asked participants to recall instances of positive influences. Whether participants have actually experienced these or whether they have reinterpreted certain experiences retrospectively remains unclear. As it is, however, the present 
findings are in line with previous research (e.g., Jones \& McAdams, 2013).

Regarding the present sample, it is unfortunate that we had to discard some participants' data because they had produced missing data in the questionnaires or did not want to answer the interview question. Thus, the sample size did not suffice to reach a power of 80 . On the other hand, the sample size is comparable to Jones and McAdams (2013). Furthermore, the present study complements theirs nicely in that they studied adults from 55 through 59 years, whereas we report findings from participants from 59 years up, thus increasing the generalizability of the finding. Of course, the same relation could also be tested in a broader age range across middle adulthood.

Finally, a more diverse (e.g., concerning sexual orientation) sample would increase the scope of the findings. In this context, ethnicity/cultural background of participants might play an important role. Positive teacher/mentor influence seems to affect the generativity development of Black and White American differentially (Jones \& McAdams, 2013). A comparable analysis could not be conducted in the present sample, as there were no participants with a migration background. Culturally diverse samples would be desirable, however, as cross-cultural research has shown differences in advice seeking, advice giving, and reactions to advice (Feng $\&$ Feng, 2018): For example, spontaneous advice giving is more common in collectivist cultures so that differences in the definition of what constitutes a positive influence might arise.

Future research might focus on potential explanations of the link between positive influences and generative concern. For example, gratitude might spawn generativity by motivating people to give something back (Froh et al., 2010). Interestingly, some non-generative people also used the norm of reciprocity, which is inherent to the idea of giving back: They argued that they did have anybody to help them, so why should they help others (Cheng et al., 2008). Given the social and individual relevance of generativity, it is important to develop ideas on how to promote generativity in those who have not experienced it themselves (see Marsaglio, 2008).

The present finding on the moderating effect of extraversion also informs generativity research with respect to how the intended recipients perceive generative efforts. Previous research has shown that it was detrimental to generative individuals' well-being when their generative efforts were rejected (e.g., Tabuchi et al., 2015). However, under what circumstances the intended recipient actually does reject generative offers needs more scientific attention. Apparently, as Thomas et al. (2021) and the present findings suggest, recipients' traits play a role in this context.
In sum, despite some limitations, the present study contributes some insights into the development of generativity. First, recalled positive influences related to generative concern but not generative behavior. Second, extraversion moderated this relation, which suggests a double role of extraversion in the development of generativity in that it is a direct predictor of generativity but also modulates the extent to which learning opportunities for generativity are taken. This opens up a new perspective on how broad personality traits affect personality development in other domains such as generativity.

\section{Appendix A}

Example 1: After my husband's death [when I was 31], the pastor of [my hometown]. I could always unburden myself of my worries there. For example, when I was not paid any welfare money, he said: "That can't be, I'll take care of that." Then he gave me an address, and eventually I received some welfare money. That's what he was like, taking care of practical matters, but also emotionally.

Example 2: I met a couple in America, with whom I lived for one-and-a-half years [when I was 19]. And they were my surrogate parents of a kind. And they have had a super positive influence on me: They were very active, politically for example. They realized what I needed to know to get along in America, because I went there a little naively and there was a lot I didn't know. But they weren't know-it-alls, you know. They took care of me and wanted me to tell them where I was going and things like that. But they did all that so very nicely and were very tolerant, and they brought me into contact with so many things that otherwise I might never have discovered for myself. And yes, they were quite some role models for me.

Example 3: I was doing an apprenticeship [when I was 17] and I couldn't swim. At my workplace, I met my mentor-if you will-and with him, I got to know the German Life Saving Association. I was so fascinated that I had to become a member, and I have stuck with it up to now. I learnt swimming, got my swimming teacher license, got involved in voluntary youth work. I enjoyed it so much that I gave my every free weekend for the Association. It was fun and it has shaped me, and I still enjoy it. Still, every Monday, I teach some five- through seven-year-olds how to swim.

Acknowledgements We thank Nils Schumacher and Markéta Künne for coding the interview responses. In addition, thank you to all student assistants involved in the data collection and interview transcription processes. Finally, we would like to express our gratitude to all participants who agreed to participate in the additional interview session. 
Funding Open Access funding enabled and organized by Projekt DEAL. This research was funded by a Grant of the German Research Foundation (HO2435/5-1)

Data Availability The data that support the findings of this study, along with code and information on additional analyses, are openly available in the Open Science Framework at https://osf.io/6yvbr.

\section{Declarations}

Conflict of interest The authors declare no conflicts of interest.

Open Access This article is licensed under a Creative Commons Attribution 4.0 International License, which permits use, sharing, adaptation, distribution and reproduction in any medium or format, as long as you give appropriate credit to the original author(s) and the source, provide a link to the Creative Commons licence, and indicate if changes were made. The images or other third party material in this article are included in the article's Creative Commons licence, unless indicated otherwise in a credit line to the material. If material is not included in the article's Creative Commons licence and your intended use is not permitted by statutory regulation or exceeds the permitted use, you will need to obtain permission directly from the copyright holder. To view a copy of this licence, visit http://creativecommons.org/licenses/by/4.0/.

\section{References}

Baron, R. M., \& Kenny, D. A. (1986). The moderator-mediator variable distinction in social psychological research: Conceptual, strategic, and statistical considerations. Journal of Personality and Social Psychology, 51(6), 1173-1182. https://doi.org/10.1037/00223514.51.6.1173

Blatný, M., Millová, K., Jelínek, M., \& Romaňákova, M. (2019). Personality predictors of midlife generativity: A longitudinal study. Journal of Adult Development, 26(3), 219-231. https://doi.org/10. 1007/s10804-018-9323-z

Busch, H., Hofer, J., Poláčková Šolcová, I., \& Tavel, P. (2018). Generativity affects fear of death through ego integrity in German, Czech, and Cameroonian older adults. Archives of Gerontology and Geriatrics, 77, 89-95. https://doi.org/10.1016/j.archger.2018.04.001

Busch, H., \& Kranz, D. (2021). Received gay generativity increases the likelihood of gay generativity by fostering gay identity affirmation [Manuscript submitted for publication]. Trier University.

Carpenter, J. C., \& Freese, J. J. (1979). Three aspects of self-disclosure as they relate to quality of adjustment. Journal of Personality Assessment, 43(1), 78-85. https://doi.org/10.1207/s15327752j pa4301_11

Cheng, T.-S., Chan, W., \& Chan, A. C. M. (2008). Older people's realization of generativity in a changing society: The case of Hong Kong. Ageing and Society, 28(5), 609-627. https://doi.org/ 10.1017/S0144686X07006903

Cox, K. S., Wilt, J., Olson, B., \& McAdams, D. P. (2010). Generativity, the Big Five, and psychosocial adaptation in midlife adults. Journal of Personality, 78(4), 1185-1208. https://doi.org/10.1111/j. 1467-6494.2010.00647.x

Donnellan, M. B., Oswald, F. L., Baird, B. M., \& Lucas, R. E. (2006). The Mini-IPIP scales: Tiny-yet-effective measures of the Big Five factors of personality. Psychological Assessment, 18(2), 192-203. https://doi.org/10.1037/1040-3590.18.2.192

Dougherty, T. W., Cheung, Y. H., \& Flora, L. (2007). The role of personality in employee developmental networks. Journal of
Managerial Psychology, 23(6), 653-669. https://doi.org/10.1108/ 02683940810894738

Eaton, L. G., \& Funder, D. C. (2003). The creation and consequences of the social world: An interactional analysis of extraversion. European Journal of Personality, 17(5), 375-395. https://doi. org/10.1002/per.477

Erikson, E. H. (1963). Childhood and society (2nd ed.). Norton.

Erikson, E. H., \& Erikson, J. M. (1997). The life cycle completed: Extended version with new chapters on the ninth stage of development. Norton.

Faßbender, K., Wiebe, A., \& Bates, T. C. (2019). Physical and cultural inheritance enhance agency, but what are the origins of this concern to establish a legacy? A nationally-representative twin study of Erikson's concept of generativity. Behavior Genetics, 49(2), 244-257. https://doi.org/10.1007/s10519-018-9943-x

Feng, B., \& Feng, H. (2018). Advice across cultures. In E. L. MacGeorge \& L. M. Van Swol (Eds.), The Oxford handbook of advice (pp. 381-400). Oxford University Press.

Fisher, B. J. (1995). Successful aging, life satisfaction, and generativity in later life. International Journal of Aging and Human Development, 41(3), 239-250. https://doi.org/10.2190/ HA9X-H48D-9GYB-85XW

Frensch, K. M., Pratt, M. W., \& Norris, J. E. (2007). Foundations of generativity: Personal and family correlates of emerging adults' generative life-story themes. Journal of Research in Personality, 41(1), 45-62. https://doi.org/10.1016/j.jrp.2006.01.005

Fritz, M. S., \& MacKinnon, D. P. (2007). Required sample size to detect the mediated effect. Psychological Science, 18(3), 233-239. https://doi.org/10.1111/j.1467-9280.2007.01882.x

Froh, J. J., Bono, G., \& Emmons, R. (2010). Being grateful is beyond good manners: Gratitude and motivation to contribute to society among early adolescents. Motivation and Emotion, 34(2), 144157. https://doi.org/10.1007/s11031-010-9163-z

Grossman, M. R., \& Gruenewald, T. L. (2020). Failure to meet generative self-expectations is linked to poorer cognitive-affective wellbeing. The Journals of Gerontology: Series B, 75(4), 792-801. https://doi.org/10.1093/geronb/gby069

Gruenewald, T. L., Liao, D. H., \& Seeman, T. E. (2012). Contributing to others, contributing to oneself: Perceptions of generativity and health in later life. The Journals of Gerontology: Series B, 67(6), 660-665. https://doi.org/10.1093/geronb/gbs034

Grych, J., Taylor, E., Banyard, V., \& Hamby, S. (2020). Applying the dual factor model of mental health to understanding protective factors in adolescence. American Journal of Orthopsychiatry, 90(4), 458-467. https://doi.org/10.1037/ort0000449

Hart, H. M., McAdams, D. P., Hirsch, B. J., \& Bauer, J. J. (2001). Generativity and social involvement among African Americans and white adults. Journal of Research in Personality, 35(2), 208-230. https://doi.org/10.1006/jrpe.2001.2318

Hayes, A. F. (2015). An index and test of linear moderated mediation. Multivariate Behavioral Research, 50(1), 1-22. https://doi.org/10. 1080/00273171.2014.962683

Hayes, A. F. (2018). Introduction to mediation, moderation, and conditional process analysis: A regression-based approach (2nd ed.). Guilford Press.

Hofer, J., Busch, H., Au, A., Poláčková Šolcová, I., Tavel, P., \& Tsien Wong, T. (2016). Generativity does not necessarily satisfy all your needs: Associations among cultural demand for generativity, generative concern, generative action, and need satisfaction in the elderly in four cultures. Developmental Psychology, 52(3), 509-519. https://doi.org/10.1037/dev0000078

Hofer, J., Busch, H., Chasiotis, A., Kärtner, J., \& Campos, D. (2008). Concern for generativity and its relation to implicit power motivation, generative goals, and satisfaction with life: A cross-cultural investigation. Journal of Personality, 76(1), 1-30. https://doi.org/ 10.1111/j.1467-6494.2007.00478.x 
Jones, B. K., \& McAdams, D. P. (2013). Becoming generative: Socializing influences recalled in life stories in late midlife. Journal of Adult Development, 20(3), 158-172. https://doi.org/10.1007/ s10804-013-9168-4

Kessler, E.-M., \& Staudinger, U. M. (2007). Intergenerational potential: Effects of social interaction between older adults and adolescents. Psychology and Aging, 22(4), 690-704. https://doi.org/10.1037/ 0882-7974.22.4.690

Korte, J., Bohlmeijer, E. T., Cappeliez, P., Smit, F., \& Westerhof, G. J. (2012). Life review therapy for older adults with moderate depressive symptomatology: A pragmatic randomized controlled trial. Psychological Medicine, 42(6), 1163-1173. https://doi.org/10. 1017/S0033291711002042

Kotre, J. (1984). Outliving the self: How we live on in future generations. Norton.

Lawford, H. L., Doyle, A.-B., \& Markiewicz, D. (2013). The association between early generative concern and caregiving with friends from early to middle adolescence. Journal of Youth and Adolescence, 42(12), 1847-1857. https://doi.org/10.1007/ s10964-012-9888-y

Love, N., Nelson, G., Pancer, S. M., Loomis, C., \& Hasford, J. (2013). Generativity as a positive mental health outcome: The long-term impacts of Better Beginnings, Better Futures on youth at ages 18-19. Canadian Journal of Community Mental Health, 32(1), 155-169. https://doi.org/10.7870/cjcmh-2013-012

Marr, C., Vaportzis, E., Dewar, M., \& Gow, A. J. (2020). Investigating associations between personality and the efficacy of interventions for cognitive ageing: A systematic review. Archives of Gerontology and Geriatrics. https://doi.org/10.1016/j.archger.2019.103992

Marsaglio, W. (2008). Men on a mission: Valuing youth work in our communities. The Johns Hopkins University Press.

McAdams, D. P. (2013). The positive psychology of adult generativity: Caring for the next generation and constructing a redemptive life. In J. D. Sinnott (Ed.), Positive psychology: Advances in understanding adult motivation (pp. 191-205). Springer.

McAdams, D. P., \& de St. Aubin, E. (1992). A theory of generativity and its assessment through self-report, behavioral acts, and narrative themes in autobiography. Journal of Personality and Social Psychology, 62(6), 1003-1015. https://doi.org/10.1037/ 0022-3514.62.6.1003

McAdams, D. P., Diamond, A., de St. Aubin, E., \& Mansfield, E. (1997). Stories of commitment: The psychosocial construction of generative lives. Journal of Personality and Social Psychology, 72(3), 678-694. https://doi.org/10.1037/0022-3514.72.3.678

McAdams, D. P., \& Guo, J. (2015). Narrating the generative life. Psychological Science, 26(4), 475-483. https://doi.org/10.1177/ 0956797614568318

McAdams, D. P., Hart, H. M., \& Maruna, A. S. (1998). The anatomy of generativity. In D. P. McAdams, \& E. de St. Aubin (Eds.), Generativity and adult development: How and why we care for the next generation (pp. 7-43). American Psychological Association.

McCrae, R. R., \& Costa, P. T., Jr. (2003). Personality in adulthood: A five-factor theory perspective (2nd ed.). Guilford Press.

Moore, S. M., \& Rosenthal, D. A. (2014). Personal growth, grandmother engagement and satisfaction among non-custodial grandmothers. Aging and Mental Health, 19(2), 136-143. https://doi. org/10.1080/13607863.2014.920302

Peterson, B. E., \& Duncan, L. E. (1999). Generative concern, political commitment, and charitable actions. Journal of Adult Development, 6(2), 105-118. https://doi.org/10.1023/A:1021620824878

Peterson, B. E., Smirles, K. A., \& Wentworth, P. A. (1997). Generativity and authoritarianism: Implications for personality, political involvement, and parenting. Journal of Personality and Social Psychology, 72(5), 1202-1216. https://doi.org/10.1037/00223514.72 .5 .1202
Peterson, B. E., \& Stewart, A. J. (1996). Antecedents and contexts of generativity motivation at midlife. Psychology and Aging, 11(1), 21-33. https://doi.org/10.1037//0882-7974.11.1.21

Pratt, M. W., Norris, J. E., Hebblethwaite, S., \& Arnold, M. L. (2008). Intergenerational transmission of values: Family generativity and adolescents' narratives of parent and grandparent value teaching. Journal of Personality, 76(2), 171-198. https://doi.org/10.1111/j. 1467-6494.2007.00483.x

Schoemann, A. M., Boulton, A. J., \& Short, S. D. (2017). Determining power and sample size for simple and complex mediation models. Social Psychological and Personality Science, 8(4), 379-386. https://doi.org/10.1177/1948550617715068

Stoltz, S., Prinzie, P., de Haan, A., van Londen, M., de Castro, B. O., \& Deković, M. (2012). Child personality as moderator of outcome in a school-based intervention for preventing externalising behaviour. European Journal of Personality, 27(3), 271-279. https://doi. org/10.1002/per.1892

Sun, J., Harris, K., \& Vazire, S. (2020). Is well-being associated with the quantity and quality of social interactions? Journal of Personality and Social Psychology, 119(6), 1478-1496. https://doi.org/ $10.1037 / \mathrm{pspp} 0000272$

Syed, M., \& Nelson, S. C. (2015). Guidelines for establishing reliability when coding narrative data. Emerging Adulthood, 3(6), 375-387. https://doi.org/10.1177/2167696815587648

Tabuchi, M., Nakagawa, T., Miura, A., \& Gondo, Y. (2015). Generativity and interaction between the old and young: The role of perceived respect and perceived rejection. The Gerontologist, 55(4), 537-547. https://doi.org/10.1093/geront/gnt135

Thomas, N., Hofer, J., \& Kranz, D. (2021). Listen to life-stories: Effects of intergenerational exchange on adolescent self-concept clarity [Manuscript submitted for publication]. Trier University.

Tov, W., Nai, Z. L., \& Lee, H. W. (2016). Extraversion and agreeableness: Divergent routes to daily satisfaction with social relationships. Journal of Personality, 84(1), 121-134. https://doi.org/10. 1111/jopy.12146

Ursachi, G., Horodnic, I. A., \& Zait, A. (2015). How reliable are measurement scales? External factors with indirect influence on reliability estimators. Procedia Economics and Finance, 20, 679-686. https://doi.org/10.1016/S2212-5671(15)00123-9

Versey, H. S., Stewart, A. J., \& Duncan, L. E. (2013). Successful aging in late midlife: The role of personality among college-educated women. Journal of Adult Development, 20(2), 63-75. https://doi. org/10.1007/s10804-013-9157-7

Watson, D., \& Hubbard, B. (1996). Adaptational style and disposition structure: Coping in the context of the five-factor model. Journal of Personality, 64(4), 737-774. https://doi.org/10.1111/j.14676494.1996.tb00943.x

Wellenzohn, S., Proyer, R. T., \& Ruch, W. (2018). Who benefits from humor-based positive psychology interventions? The moderating effects of personality traits and sense of humor. Frontiers in Psychology, 9, 821. https://doi.org/10.3389/fpsyg.2018.00821

Westermeyer, J. F. (2004). Predictors and characteristics of Erikson's life cycle model among men: A 32-year longitudinal study. International Journal of Aging and Human Development, 58(1), 29-48. https://doi.org/10.2190/3VRW-6YP5-PX9T-H0UH

Zacher, H., Rosing, K., Henning, T., \& Frese, M. (2011). Establishing the next generation at work: Leader generativity as a moderator of the relationships between leader age, leader-member exchange, and leadership success. Psychology and Aging, 26(1), 241-252. https://doi.org/10.1037/a0021429

Publisher's Note Springer Nature remains neutral with regard to jurisdictional claims in published maps and institutional affiliations. 